\title{
The Web of Augmented Physical Objects
}

\author{
Thomas Nicolai \\ University of St. Gallen \\ thomas.nicolai@unisg.ch
}

\author{
Florian Resatsch \\ University of Arts Berlin \\ resatsch@ieb.net
}

\author{
Daniel Michelis \\ University of St. Gallen \\ michelis@ieb.net
}

\begin{abstract}
This article investigates the growing complexity and connectivity between two former separated interaction spaces - the real and the virtual world. It is our attempt to augment the physical world to a world of digitally augmented objects. Moreover we are looking at real products tagged with digital communication units like embedded systems or the RFID technology. To understand these impacts we need to identify the different levels of connectivity between real and virtual worlds and therefore the relationship of a product with its digital counterpart. As a point of departure we define the terms medium and agent to get a theoretical view on the associations in the real and virtual world. We identify the digital identification of virtually enhanced products as a main part for the creation of a new networked physical world where real products are heavily connected to and with their digital counterparts.
\end{abstract}

\section{Introduction}

According to SCHMID, Medium can be referred to as interaction spaces, insofar as what the exchange, in particular the communi-cational exchange between the participating agents, makes possible. Such interaction spaces or medium require three components, if they are to be used as medium in society [16]:

- The social or organizational components define the rolls in which the interactive partners appear and the rules according to which they interact (protocol).

- The logical space comprises the common language, i.e. the characters, the laws of their construction (syntax), their meaning (semantics) and the rules of their argumentation and conclusions. Without a common logical space, the exchange of characters in code is of course possible, but not the exchange of knowledge, that makes foremost the creation of a common knowlegde and a common world possible.

- A physical component, that enables the physical interaction of the physical agents. These components can also be referred to as transfer mediums.

The goal of this first chapter is the classificational overview of the web of objects [4] as medium. The three highlighted components of medium concepts should serve as a guideline, according to which the characteristics of virtually augmented objects can be described. A medium is, according to the here applicable medium concept, an interactive space for agents. It enables the communication between agents and the transaction of objects. The function of these transports, the conveying of information objects, is the root of technical medium concepts since Claude Shannon [17]. Technical medium accomplish this goal between and beyond any time frame. They all work according to the same principal of the circuit, that as a physical medium links agents in space and/or time and enables communication. Medium are hence defined by their function: they enable the exchange, the interaction between agents (Fig. 1) [32].

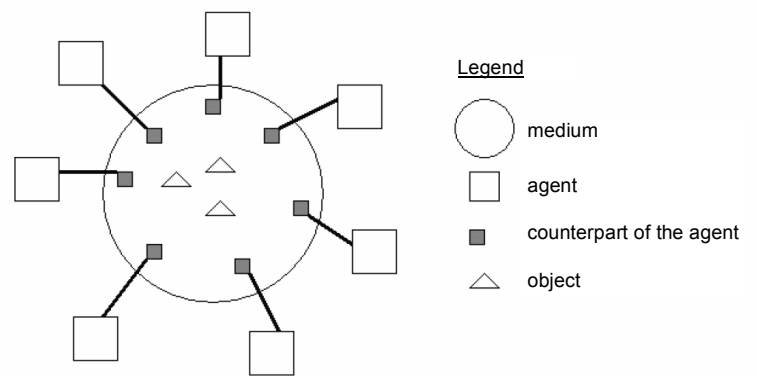

Figure 1: Medium as a sphere for agents. 
The here employed medium concept differentiates between the three itemized components, and shows that the characteristics of medium are dominated by the web of objects. In the near future, this aspect will also be integrated into the economy and in our everyday lives. The components are integrated into a social and organizational space, a logical space, and a circuitry (physical medium).

\subsection{The social and organizational space.}

The agents have only specific space/time entry points into the circuitry, in which specific situations, specific rights and duties, access and acts are executed. The entirety of these rights and duties, the negotiation of which are regulated by an agent, we refer to as the roll or the function of the agent. Furthermore, the actions of the agents in the circuitry can be linked through a control flow regulator and in a data flow or process. In the example of email protocols, there is the roll of the user, the user agent (UA) and the message transfer agent (MTA) with their respective rights and duties as well as some processes, which their interaction regulates. (i.e. connection protocol, display of an email). These rules determine the organization of the usage of media. Without them no real interaction can take place. These allow for a common understanding of the role of the other and a common knowledge of the flow of interaction. Within a medium such a world arises that is not only a world of language, but is also circumscribed by a common theme and understanding of the rights and duties of the integrated members of the society, i.e. their roles and functions.

\subsection{The logical space}

So that communication works a transport system must satisfy these conditions: the sent objects must be by both the sender and receiver understandable. They must inhabit the same interpretation space and speak the same language. Communication is defined as symbolic interaction. The symbols that are exchanged during communication are regulated in the logical space of the medium. This space allocates symbols in the sense of a (formal) language. These become, starting from an alphabet of base characters, juxtaposed characters (words, sentences, etc.) arranged according to the rules of syntax. Thus information- both descriptive factual information as well as prescriptive handling information- is presented. There are characters, with which objects are labeled and possibly presented, to which the agents react and with which they are integratedemail for example, the mail itself with its component parts like header and body, or general data like documents. In addition there are characters, which the subjects denote and allocate like names, addresses, and commands. We consider this logic space of communication in light of agents, to point out above all the development of web semantics. Decidedly, the semantic level is about the combination of different informational objects within an interaction space. Ontology is the basis for a common space of interaction.

\subsection{The channel system}

The exchange or interaction is unfolded by way of interfaces, and is based on objects (i.e. email) or the request of services (i.e. the activation of a transaction, the playing back of a song). These interfaces serve as access points to borrow a term from telecommunications. They are the service access points (SAP) of a circuit system, and comprise the physical basis of the medium. The circuit system is the physical part of the medium, and is also called the carrier medium. As we will see in the following sections, it is through the interaction of an array of technologies that a new type of circuitry is possible, one that stretches across the virtual and physical worlds. The next section describes the digital counterparts of the physical products in the virtual world and the web of objects as the accumulated number of information objects connected with and to real objects [4].

\section{The Digital Counterpart and the web of objects}

Every day we are in close contact with many objects of many kinds. Whether it is a complex entity such as the computer we use in the office or simply pen and paper. All of these objects, now referred to as products, share a rather analogue process of being planned by engineers or product designers to the time when the product is in our possession. According to WordNet2.0 a product is "an artefact that has been created by someone or some process" (WordNet2.0). This chapter considers the product as being more than a physical object (According Wikipedia), and 
describes the existence of a virtual counterpart of such an object - the "Digital Counterpart" and how the web of objects is defined by the product's digital counterpart.

\section{1 Products as physical entities}

A product is a physical entity, designed for a certain purpose or process. Consider the example of a cup. It can be used to drink coffee, but also as a design object. The cup itself does not contain any further information, it's just a cup. Although there is much information about the cup available, a customer currently buys a cup and uses it. No further information is shared or attached directly to the product although the product definition might be interesting and useful information in some situations. The product definition is an essential premise for successful product design to capture and understand customer needs effectively and subsequently transfer them into design specifications [15]. Usually a design team explores the customer needs and the company's related technological capabilities, concluding the design process with a definition of the product [19]. This product definition is represented as a list of requirements, specifications and target values, depending on the complexity of the product. The information is a mix of qualitative and quantitative descriptions of the product. A company records important information on the product and the productions process for future reference and produces formal documents that may undergo many amendments along with scrutiny [18]. Some products keep more important information inherit to it than others, for example pills to cure heart diseases compared with a simple pen. All the available information could be shared with the customers and interested persons. The following figure shows the current situation with products:

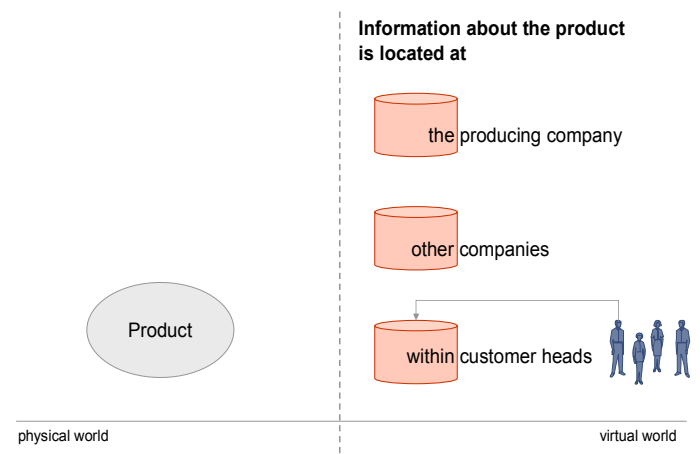

Figure 2: The current situation
The product is located in the real world, called the physical world. In recent times digital products have arisen which cannot be touched and are only available in digital form. According to [10], a digital product can be defined as followed: 1. It is "digital", the digital product consists of bits and bytes and 2. a digital product can be obtained through electronic channels such as the Internet. For the purpose of this paper, we concentrate on the real life products in the physical world when we speak of products. In the virtual world, information about the product is stored in many different locations. For example, the company that produces the product stores a lot of product related data in the company databases, the aforementioned product definition for example. Other companies such as advertising companies may store different data about the product, maybe the product usage as described in an advertising campaign. Even more data is available in the heads of the customers who daily deal with the product and keep the hugest knowledge base on the product's attributes. Although this knowledge might be difficult to gather, it is a possible source of information about the product, its advantages and usages. All the information combined, generates what we call "the digital counterpart" - the digital representation of the product - a possible description of all necessary data on the product which in total gives a detailed picture of the product itself. The following figure shows the digital counterpart.

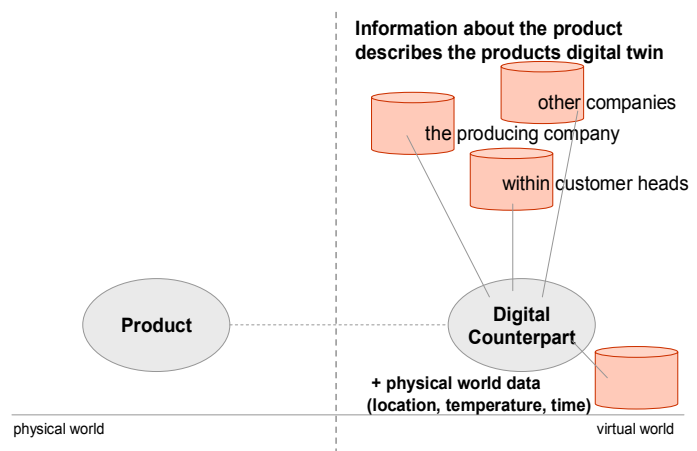

Figure 3: The digital counterpart

\subsection{The virtual augmented object}

Still, the physical world is separated from the virtual world and all the information within it. Now, with technologies such as radio frequency identification (RFID), the product can be digitally augmented if the 
digital data is attached to it, for example, by sticking a RFID tag or integrating an embedded system. Attaching a digital tag enables the connection of the product to the virtual world by either storing information on the chip itself or storing a link to the internet which can be used by mobile devices as the premier source of information. The object - product plus digital counterpart connected to the product generates the virtual augmented product sphere with an augmented object in the centre. Similar to the early 90s approaches from Wellner [22,23], Fitzmaurice [7], and Ishii [12], Want et al. [5] describe several possibilities of augmented products, everyday tools and objects. Building invisible interfaces, Want et al. also show that humancomputer interaction will be based on a screen-less level in the future as stated by Lev Manovich [13]. Want et al. augmented books, documents, as well as business cards and a wrist watch. In this scenario, a tag is embedded in a wristwatch. When the user brings the wristwatch close to an active zone of a personal computer a calendar application for that particular user is shown for the current day, at the current time. The wristwatch behaves in all other respects exactly as it normally would [5]. Therefore this wrist watch is augmented by the tag and provides a further functionality to the user. The computer used as the device to show the calendar is also augmented in its functionality since the interface to open the calendar application works without a mouse or fingers.

\subsection{The virtual augmented product sphere}

Figure 3 shows the virtual augmented product sphere, defined by the combination of the physical and virtual world. The shown data is a possible frame for useful information on a product. The contextual data contains actual information on the location and the context of the product, if available. The current temperature, the location itself, the surrounding products, etc. enables context-aware services. Furthermore the functional data tells the user how to use the product, what the main components are, which additional products are available along with guarantee files. The formal data might show some interesting figures on the product, for example statistics. The knowledge on the production process is stored in the production data. Combining all this information, the product is augmented through the virtual augmented product.

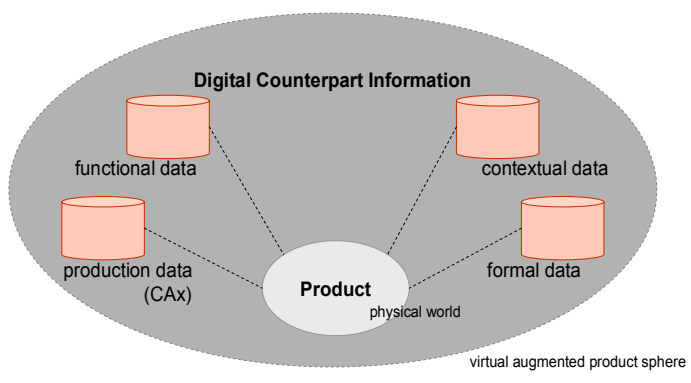

Figure 4: The virtual augmented product sphere

If all products would hold that much information, and would be interconnected to each other (The thinking fridge for example), the "real and augmented web of objects" is generated as shown in figure 4:

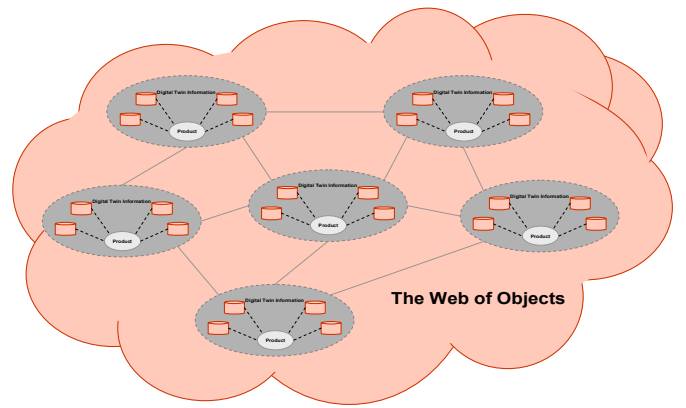

Figure 5: The web of augmented physical objects

This web of augmented physical objects is described in the next section. The section 3 takes a deeper look inside the technical and conceptual origin of the web of objects or as it could be called as well - the web of augmented physical objects.

\section{The web of augmented physical objects}

Distributed, dynamic and at the same time adaptive [5] and the so called embedded computer systems [2] support and broaden more and more our possibilities for communication and the very systems that operate discretely and more powerfully in the background. Every physical object becomes an agent through the closure of a gap in the medium between real and virtual worlds, or a communications partner in the interactive space of the internet as well as through newly arising human interfaces i.e. from the research field of augmented reality [24]. These broadened virtual worlds of the internet via RFID and so called embedded computer systems are at just the beginning of a future [20,21,9]. From a technological 
perspective the following figures explain the possibilities of the connection between physical products and their digital counterparts as described in the previous section. This paragraph tries to model the theoretically described web of augmented physical objects into a technical specification. Figure 6 shows the two possible connections from the physical to the virtual world.

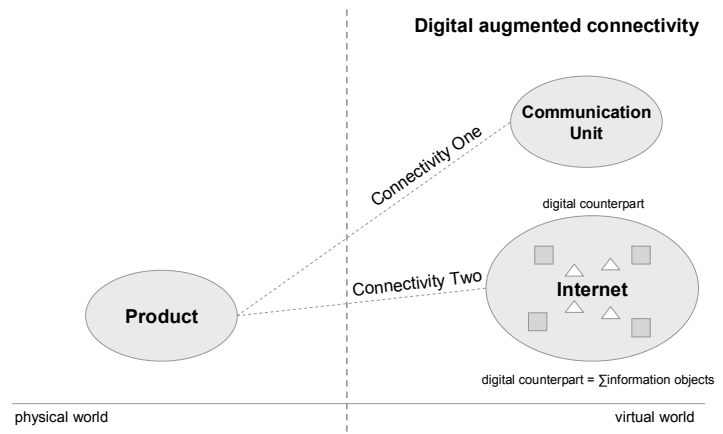

Figure 6: Two connections to the virtual world.

\subsection{Connectivity One}

Connectivity One designates the physical connections to real products with digitally identifiable communication units [6]. As designated ,tagged' products, these can also either be coupled with RFID technology or embedded systems. RFID tags are characterized through particularly simple and concentrated structures. Whereas embedded systems can operate almost the entire functional range of a normal computer system and therefore process more complex communications as well as initiate commands. RFID is the extended development of a technology that has been around already for decades. Because of the sinking prices interest in the technology has expanded. The possible alternating exchange of data with RFID over cableless, limited distance, forms, in our view, the principle of the linking of products with their digital counterparts. Thus the identification of digitally augmented products through a simple world wide designation (also called Global Unique ID - GUID) becomes fundamentally interesting. For example, the electronic product code (EPC) of the auto-ID centres [8], similar to how the internet makes a unique identification possible with IP or MAC addresses. Through a GUID it is possible to trace a linked location with information objects, over an Object Name Server (ONS) or a Domain Name Server (DNS) system $[1,14]$, to distributed media databanks or agents. The goal of this architecture is to centrally maintain the location data as well as resources for linked information. If one were to save these directly on the digitally augmented products, one would have to update, for example by adding a new information resource, the information resources of more than a hundred or possibly thousands of products. This would also apply if one were to attempt to save all of the digital counterparts of the product to the product itself. The date of the data is would not only be different from product to product, the amount of data would also be too big, based on the limited, minimized hard drives of such systems. By embedded systems, everything is about the surrounding computer systems that perform far reaching tasks in the background $[25,8]$. Already feature-embedded systems allude to larger kinds of systems that use, where required, more memory and where the system is based on the same hardware as the computer. The only difference is the limited size conditions that are required by the tight bundling of hardware and software [25]. To clarify this, we give the example of the ever present existence of this kind of system. When a coffee machine regulates the strength and amount of the coffee, an embedded system is used since an RFID system could display the temperature of the water, but not read the amount of coffee that is used. Therefore, the system broadens not only the web of objects, it allows for the changing of the product's operating state according to the previous information from the differing information objects. In addition the system would be capable of sending the average used amount of coffee to the manufacturer and maybe carry out a product adjustment. The user could be informed about new products after the coffee machine reaches it average life span. The coffee machine becomes thus another agent in the physically expanded interactive space of the web of objects.

\subsection{Connectivity Two}

„Connectivity Two" designates the contact free communication between the digitally augmented product and the internet. Accessing the virtual communication interface system to the internet is based of the Ethernet and diverse wireless technologies like WLAN, Bluetooth and UMTS. This system may be designated as agent as it was described in the first section. The following figure attempts to clarify the relationships and details of „Connectivity Two“. 


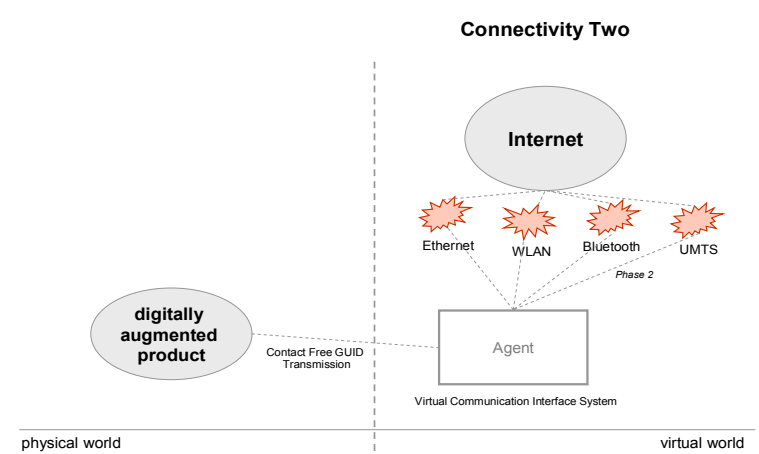

Figure 7: Connectivity Two

A detailed consideration of the figure reveals the difference between the first communication phase of the digitally augmented products and the agents, as well as a second phase of an agent communicates with additional internet based agents. The first connection between real and virtual world actually takes place here. On the one hand these can be connected with a sub computer through the RFID reading machine or as the case may be linked to a server system or directly into an embedded system if such an agent is already embedded in the system.

\subsection{The virtual communication interface system}

The discovery and merging of information sources and objects is a savvy approach to the digital broadening of products. In this way the process of identifying information resources is initiated through the ,tagged" product and the forwarding of GUID to the agents. After the successful identification via a DNS or ONS similar system, the digital information objects can be traced through the communication with the agents. These can in turn, depending on the context, become the digital counterpart, with which the agents can be merged (see the following figure).

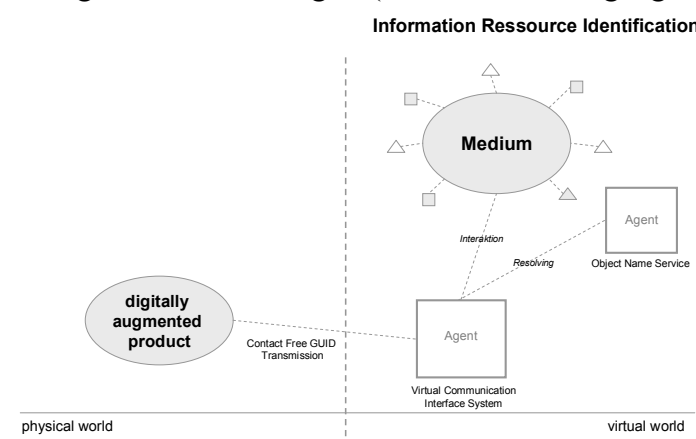

Figure 8: Information Resource Identification
The identification of information resources occurs via the resolution of the GUID in individual Internet resources. These resources can be here for example web site or also semantic web services, offered from different agents [1]. These become identified in the interactive space of the medium of the internet [16], whose information objects in turn convey the already accommodated dependence of the previous interaction - the web of the augmented physical objects is real.

\section{Conclusion}

The internet develops more and more into a truly expanded medium, wherein many technological and organizational limits are to be researched. In this article we have attempted to present through an analysis of the concepts medium and agent a fundamental understanding of previous definitions of the digital broadening of physical products via digital counterparts. Enhancing a physical product with digital information objects can lead to completely new creations and process chains. The creation of digital objects allows broadening the previously virtually-limited interactive frame of the internet and its agents i.e. via technologies like RFID and Web services. Through these new connections it will become possible to carry into the real world what was known before only in the virtual world of - the expansion of the internet into a smart.

Real and virtual worlds emerge into a new expanded interactive space where the medium is expanded in this way that physical products carry into agents that are from now on a part of this interactive space. Components like RFID are the first step to open real objects or like we defined before real products the way into the digital space. Therefore we tried to develop a connectivity concept which shows the minimum requirements to get one step closer to the Web of Augmented Physical Objects and according to SCHMID the tree components of medium as a point of departure for our common understanding. As we can say there is neither currently an implementation nor a prototype existing in this field of research. Next step in the research area could be to develop a system like this and to show with a prototype the limitations of this approach. Furthermore it could be explored which products can be turned into agents and what would be the economical values of this. 


\section{References}

[1] Auto-ID Center, Auto-ID Savant Specification 1.0 (2003). http://www.eannet-

france.org/download/nonprotege/b_outils ean/rfid/rfid ne w/WD-savant-1_0-20030911.pdf, Accessed 2005-02-15

[2] Beigl, M., Gellersen, H. Smart-Its: An Embedded Platform for Smart Objects.

http://www.grenoble-soc.com/proceedings03/Pdf/62beigl.pdf, Accessed 2005-02-02

[3] J. Bohn and F. Mattern (2004), Super-Distributed RFID Tag Infrastructures.

http://www.vs.inf.ethz.ch/publ/papers/bohn_sdri_2004.pdf, Accessed 2005-02-21

[4] Brickley, D. (1999), Semantic Web History: Nodes and Arcs 1989-1999.

http://www.w3.org/1999/11/11-WWWProposal/, Accessed 2005-02-27

[5] Distributed Software Engineering Group Research into Ubiquitous Computing.

http://www-dse.doc.ic.ac.uk/Research/ubicomp.html, Accessed 2005-02-27

[6] Engels, W. D. et al (2001), The Networked Physical World: An Automated Identification Architecture.

http://csdl.computer.org/comp/proceedings/wiapp/2001/113 7/00/11370076.pdf, Accessed 2005-02-05

[7] Fitzmaurice, George: Situated Information Spaces and Spatially Aware Palmtop Computers, CACM, 36(7), July 1993, pp.38-49.

[8] Flörkemeier, Cristian (2004): Die

Technologiestandards des Auto-ID Centers.

http://www.vs.inf.ethz.ch/publ/papers/floerkem-autoid2004.pdf, Accessed 2005-02-28

[9] In-Stat (2005): RFID Tag Market to Approach \$3 billion in 2009.

http://www.instat.com/newmk.asp?ID=1206, Accessed 2005-03-04

[10] Klose, Martina (2002): Design Patterns for Digital Products in the Digital Economy.

http://www.unisg.ch/www/edis.nsf/wwwDisplayIdentifier/2 628/\$FILE/dis2628.pdf, Accessed 2005-03-01

[11] Barvick, Scott (2004): [Rfid] Working group charter info strawman.

http://www1.ietf.org/mail-

archive/web/rfid/current/msg00002.html, Accessed 200503-03
[12] Isbii, H. and Ullmer, B.: Tangible Bits: Towards Seamless Interfaces between People, Bits, and Atoms. Proceedings of CHZ'97, pp. 234-241

[13] Manovich, Lev 2001: The Language of New Media, MIT Press, Cambridge, Mass.

[14] Mockapetris, Paul (1987): DOMAIN NAMES CONCEPTS AND FACILITIES (RFC1034).

http://www.ietf.org/rfc/rfc1034.txt, Accessed 2005-01-17

[15] Pugh, S. 1991. Total Design: Integrated Methods for Successful Product Engineering, Wokingham Mass., Addison-Wesley.

[16] Schmid-Isler, Salome: Konzepte des Beat Schmid, Working Paper, Institute for Media and Communication Management, University of St. Gallen, 2004

[17] Shannon, C. and Warren, W. 1963: Mathematical Theory of Communication. University of Illinois Press, Illinois.

[18] Tseng, M., Jiao, J: Computer-Aided Requirement Management for Product Definition: A Methodology and Implementation, in: Concurrent Engineering: Research and Applications Vol.6, No.3, June 1998, pp.145-160

[19] Ullman, D.G. 1992: The Mechanical Design Process. McGraw Hill.

[20] Venture Development Corporation: DEVELOPMENT TOOLS GROW EXPLOSIVELY WHILE MOST OTHERS ENJOY LACKLUSTER RESULTS.

http://www.vdc-

corp.com/embedded/press/archives/99/pr99-22.html, Accessed 2005-02-23

[21] Venture Development Corporation: RFID IN THE SUPPLY CHAIN

http://www.vdc-corp.com/autoid/white/04/walfactor2.pdf, Accessed 2005-02-23

[22] Wellner, Pierre: Tactile Manipulation on the DigitalDesk. Video in CHI'92 Special Video Program, ACM SZGGZUPH Video Review 79

[23] Wellner, Pierre, Interacting with paper on the Digital Desk, CACM, 36(7), July 1993, pp. 86-96.

[24] Wellner, Pierre et al. (1993) Computer-Augmented Environments: Back to the real world. Special Issue of the Communications of the ACM, vol. 36, no. 7, July 1993

[25] Wikipedia Definition von embedded System. http://de.wikipedia.org/wiki/Embedded_Systems, Accessed 2005-03-01 